\title{
Aportaciones teóricas significativas sobre el liderazgo carismático y transformacional
}

\author{
Ganga Contreras, Francisco* \\ Navarrete Andrade, Erwin**
}

\section{Resumen}

En los últimos años han emergido profusas publicaciones que contribuyen a comprender el liderazgo transformacional y carismático, todas las cuales han provocado la germinación de diferentes teorías, enfoques y modelos que explican este fenómeno desde ópticas y contextos diversos. Considerando esta realidad, este trabajo tiene como finalidad, realizar una revisión de las contribuciones teóricas más relevantes sobre el liderazgo carismático y transformacional. Para ello, se han utilizado fuentes secundarias (revisión documental), efectuando primordialmente una auscultación de las principales publicaciones teóricas y empíricas de mayor impacto en revistas indexadas (primordialmente en ISI, Scopus y Scielo), como también, algunos libros de la especialidad. Entre los hallazgos más sustantivos, es posible destacar lo que ocurre con el liderazgo tranformacional y el carismático, mientras el primero influye preponderantemente en el ámbito de cultura o el desarrollo organizacional; el segundo coloca su foco en el líder auténtico, el cual se visualiza como una figura espiritual; sin embargo, las ópticas se complementan y no es factible afirmar que uno es mejor que otro, debido a que muchos piensan que el carisma es consustancial e intrínseco del liderazgo transformacional.

Palabras clave: Liderazgo transformacional, liderazgo carismatico, liderazgo, administración de organizaciones.

Recibido: 20-01-12 Aceptado: 10-03-14

* Administrador Público, Licenciado en Administración, Magíster en Administración de Empresas, DEA en Organización de Empresas, Doctor en Administración de Empresas y Postdoctorado en Ciencias Humanas. Actualmente es académico jornada completa del Departamento Ciencias del Desarrollo de la Universidad de Los Lagos-Campus Santiago. Correo Postal: República №517, Universidad de Los Lagos, Santiago, Chile. E-mail: fganga@ulagos.cl

** Investigador Asociado Universidad Autónoma de Chile, Chile. Académico de la Universidad Central de Chile, Escuela de Industrias. E-mail: erwineconomy@hotmail.com 


\title{
Significant Theoretical Contributions regarding Charismatic and Transformational Leadership
}

\begin{abstract}
In recent years, profuse publications that contribute to understanding transformational and charismatic leadership have emerged, all of which have led to the germination of different theories, approaches and models that explain this phenomenon from diverse contexts and viewpoints. The purpose of this article is to review the most relevant theoretical contributions about charismatic and transformational leadership. To achieve this, secondary sources were used (a literature review), primarily an auscultation of the main theoretical and empirical publications of greatest impact taken from indexed journals (mainly ISI, Scopus and SciELO) as well as some specialized books. Among the essential findings, it is possible to highlight what occurs with transformational and charismatic leadership. While the former affects predominantly the field of culture or organizational development, the second places its focus on the authentic leader, visualized as a spiritual figure. However, these viewpoints complement each other, and is not feasible to claim that one is better than the other, because many people think that charisma is inherent and intrinsic to transformational leadership.
\end{abstract}

Keywords: Transformational leadership, charismatic leadership, leadership, administration of organizations.

\section{Introducción}

Estudiar el fenómeno del liderazgo, siempre resultará tremendamente necesario y atractivo, dado que nadie podría discutir que el éxito organizacional depende prioritariamente de las verdaderas y acreditables competencias que tengan los sujetos humanos que cumplen funciones directivas o de jefaturas. Los retos que tienen estos personajes son cada vez más enrevesados y desafiantes, pues junto con los conocimientos que se les demandan, deben tener una serie de cualidades como: habilidades comunicacionales (capacidad de oratoria), comportamientos equilibrados (emocionalmente estables), inspiradores de respeto y confianza, templados, prudentes, vigorosos (fuertes y con actitud positiva) y altamente motivadores; de modo tal que encaminen a su colaboradores hacia el logro de las metas organizacionales. Esto significa que a un líder se le demanda idoneidad y suficiencia para codificar y decodificar la misión y visión institucional en actuaciones concretas, precisas y no troceadas.

Podría afirmarse entonces, que el interés del mundo académico por esta línea disciplinaria, estriba en las transformaciones radicales y veloces que está experimentado la sociedad en términos, valóricos, cívicos, educacionales e incluso bélicos, tal como lo expresaba Bass en el año 1999. Estos acontecimientos demandan preceptivamente formas novedosas y singulares de dirección, focalizadas en desarrollar por ejemplo: el compromiso, la confianza, el respeto, el aprecio y la lealtad de los seguidores hacia el líder.

Como se sabe, en los últimos años, han surgido copiosos estudios que abor- 
dan estas materias, suceso que ha derivado en el emergimiento de un ubérrimo número de teorías, enfoques y modelos que intentan elucidar estas temáticas. Una de las perspectivas que ha conquistado mucho terreno, dice relación con el liderazgo carismático y el liderazgo transformacional; en este sentido Yukl y Van Fleet indicaban el año 1992, que estas ópticas consideran los marcos teóricos más utilizados a nivel de investigación científica en el ámbito de la administración de organizaciones (teoría de los rasgos del líder, poder, conducta y variables situacionales); y por lo mismo, podría decirse que son altamente confiables y preconizables.

Tomando en consideración las argumentaciones y disquisiciones previas, se ha planteado como propósito central de esta indagación, realizar una revisión documental relacionada con los aportes teóricos más relevantes que hacen referencia al liderazgo carismático y transformacional.

Para abordar lo anteriormente señalado, se utilizó una metodología de recolección de datos basada en fuentes secundarias (revisión hemerográfica), lo que implicó revisar y analizar libros y revistas especializadas, esencialmente indexadas en las bases de datos ISI (Institute for Scientific Information), Scopus (Elsevier) y Scielo (Scientific Electronic Library Online).

En cuanto al contenido, se efectúa, en primera instancia, una conceptualización del concepto de liderazgo propiamente, para luego, realizar un acercamiento al concepto de liderazgo y liderazgo carismático. Seguidamente, se desarrolla un trabajo similar con el duplo liderazgo transformacional; acto seguido, se realizan algunas indagaciones contemporáneas, que han abordado estos tópicos; para finalizar con una sucinta presentación de las diferencias y afinidades entre los líderes carismáticos y los transformacionales.

\section{Adyacencia al concepto de liderazgo carismático}

Antes de acercarnos al concepto de liderazgo carismático, se considera útil y pertinente, el tener un arrimo a la conceptualización de liderazgo. Al respecto se puede señalar que existen múltiples y profusas definiciones, que seguramente posibilitarían la escritura de varias paginas o una alfaguara de libros, pero utilizaremos algunas que permitan tener una especie de "panóptica" general. En este orden de cosas, Certo (2000: 326), quien citando a Goldman (1998), sostiene que el liderazgo es el proceso de dirigir la conducta de otros hacia el alcance de algún objetivo; en ese sentido, significa hacer que las personas actúen de cierta manera o sigan un rumbo particular, idealmente este rumbo es coherente con factores como las políticas establecidas, por la organización, los procedimientos y la descripción de las funciones; en otras palabras, señalan que el tema central del liderazgo es lograr que se alcancen las cosas, por medio de la gente.

A su vez House et al (2002), expresan que el liderazgo se asocia al proceso por medio del cual, un individuo ejerce la capacidad para influir y motivar a seguidores, de tal forma que éstos puedan aportar a los propósitos establecidos y al alcance del éxito del proyecto que tiene la organización. 
Complementariamente, Fernández $(2002,19)$ se indica que el ejecicio implica gestionar adecuadamente el cambio, fijar el rumbo de la organización, definir la cultura, motivar e inspirar y alinear a la gente. La anterior afirmación implica considerar a los líderes como verdaderos agentes de cambio, es decir, personas cuyos actos afectan a otras personas moviéndolas hacia un punto determinado (Ganga, 2013:55).

A su vez Casado (2003: 175) cree que el liderazgo es un proceso dinámico y complejo de influencia para alcanzar objetivos mediante una eficaz combinación de recursos y personas en una situación concreta. Una definición suplementaria, es aquella que expresa que el liderazgo puede definirse como la capacidad y el proceso mediante el cual un individuo influye sobre la conducta de los demás con la finalidad de alcanzar los obejtivos propuestos (Pirela et al., 2004).

Una mirada un tanto distinta tienen Antonakis et al (2004), que postulan que el liderazgo es primordialmente un procedimiento atributivo (basado en la cualidad o atributo de un sujeto), resultado de un proceso de percepción social, siendo la esencia del mismo el ser apreciado y calificado como líder por los otros.

Por su parte, Robbins y De Cenzo (2008, 247), tienen una definición bastante completa, y comienzan por indicar que el liderazgo es la habilidad que se demuestra cuando se influye en los demás para actuar de cierta manera. Los autores agregan, que mediante la dirección, el aliento, la sensibilidad, la preocupación y el apoyo se inspira a los seguidores para que acepten retos y logren metas que podrían percibirse como difíciles o inasequibles. Finalizan señalando que ser líder significa construir compromiso de lograr una meta entre los que son liderados, así como un fuerte deseo en ellos de seguirlo.

Finalmente Ramirez (2012: 92) expresa que el liderazgo tiene elementos claves como el sentido de la dirección, trabajo en equipo, inspiración, ejemplo y aceptación por parte de los demás.

A partir de los basamentos previos, se estima que se está en condiciones de avanzar un paso más adelante e intentar comprende el alcance del concepto de carismático.

Como se sabe, dentro de los tipos de liderazgo que generalmente llaman mucho la atención, está el liderazgo que utilizan determinadas personas, a partir de sus peculiaridades o características personales especiales, conocidas como carisma. Habitualmente a esta modalidad se le conoce también como "liderazgo motivacional" o de "influencia idealizada".

Una primera contigüidad útil para introducirnos en el tema, se puede encontrar en el Diccionario de la Real Academia Española (RAE, 2014), en la cual se indica que carisma, proviene de la voz latina charisma, y este del griego $\chi \square \rho 1 \sigma-$ $\mu \alpha$, de $\chi \alpha \rho \iota \zeta \varepsilon \sigma \theta \alpha 1$, que significa: agradar, hacer favores. Reconoce dos acepciones a saber:

- Especial capacidad de algunas personas para atraer o fascinar.

- Don gratuito que Dios concede a algunas personas en beneficio de la comunidad.

Adicionalmente, se puede indicar que se estima que la palabra "carisma" podría remontarse a la antigua filosofía 
griega y se refleja en el apotegma helénico "conócete a ti mismo", inscrito en el fontispicio del Templo de Apolo en Delfos, el que probablemente significaba "conoce tu condición humana", o como decía Socrátes "conocerse a si mismo es el principio de todo conocimiento, pues las verdades están en ti" y agregaba "esto no es un mandato, sino un saludo del Dios", hecho que le da evidentemente una condición espiritual y/o ascética (Parke y Wormell, 1956; Choza, 1993; Sampson, 1997; Alvira, 2000; Freixa, 2003; Masciotra 2005; Fierro 2005; Fernández 2006; Vignale 2012). Del mismo modo, el origen etimológico de la palabra auténtico -asociado a carisma- puede atribuirse a la palabra griega, authento, cuyo significado es "tener el poder total" (Trilling, 1972), lo que refleja la noción de funcionamiento auténtico mediante el cual un individuo es "el maestro de su propio ser", que tiene conciencia de si mismo y actúa con transparencia y ética; siendo por lo tanto confiable y genuino (Kernis y Goldman, 2006; Moriano et al, 2011; Gil, 2011; GarcíaCalvo, 2012).

Se podría complentar entonces, que el carisma está constituído por una serie de características y cualidades llamativas y auténticas, que hacen único, excepcional y extraordinaria a una persona; es decir, se está hablando de condiciones que le posibiltan al sujeto generar atractividad y magnetismo, posición que le permite cautivar de forma natural a sus seguidores. Como se trata de peculiaridades muy especiales, se dice que el carisma es connatural o innato; hecho que complejiza el liderazgo, pues implicaría que no se puede adquirir, sin embargo, muchos autores creen que existen ciertas prácticas asociadas a este estilo que se pueden entrenar y por lo tanto, aprender.

Para el profesor emérito de la Facultad de Administración de la Universidad de Binghamton (Universidad Estatal de Nueva York) y miembro de la Academia de profesionales de alto nivel en Eckerd College en Florida, Dr. Bernard Bass -considerado uno de los gurúes del tema dado que durante su carrera publicó alrededor de cuatro centenas de artículos en diferentes revistas- el liderazgo carismático tiene que ver con la apreciación que el seguidor tiener del líder, en el sentido de que considera que éste posee un "regalo de inspiración divina" y de que es (de alguna forma) único, insuperable y más eminente que nada en la vida y por lo mismo, no solamante confían en él, sino que lo respetan, lo veneran y lo adoran, llegando incluso a idolatrarlo, pues le atribuyen condiciones espirituales especiales (Bass, 1985).

Lo anteriormente explicado es ratificado por Molero (1995, 44-45), quien expresa que la palabra carisma proviene del griego y significa "gracia" o "don divino" -continúa señalando- que Max Weber la tomó prestada del cristianismo primitivo y la utiliza para denominar a uno de los tres tipos de dominación legítima, que según él pueden darse en la sociedad: la dominación carismática, la racional y legal y la dominación tradicional.

A su vez, Castro y Lupano (2005: 92) estiman que el líder carismático actúa sintetizando la información del medio, donde su propio sistema de valores se vuelve un organizador importante para los seguidores.

Existen dos autores, que también pueden ser considerados consolidados en 
estas temáticas, y por lo tanto no se pueden dejar al mangen de las definiciones, la referencia es Conger y Kanungo (1987) quienes consideran a los líderes carismático como "héroes" o "paladines", que son capaces de mostrar conductas extraordinarias o fuera de lo común, es decir, no convencionales y tienen, por lo tanto, capacidades especiales para transformar a las personas y encantarlas al punto de lograr compartir plenamente los propósitos que se han planteado. Estos autores, precisan que el carisma es un fenómeno atribucional, pues los seguidores le adjudican cualidades especiales al líder, basadas en las observaciones de su conducta y en los resultados asociados con su manera de ser; cuestiones o características que los transforman en verdaderos forofos de su superior jerárquico.
Como complementación, en la Figura 1 , se presenta una especie de diagrama sinóptico que muestra la forma como el seguidor visualiza a su líder, y las manera como actúan los líderes considerados carismáticos.

\section{Liderazgo transformacional}

Es perfectamente factible afirmar que el enfoque del liderazgo transformacional de Bass (1985 y 1998) es probablemente una de las teorías más influyentes, bienquistas y estudiadas hasta nuestros días (Yammarino et al., 2005). Se define en términos comportamentales, a partir de los efectos del líder sobre sus seguidores; estos últimos, desarollan un sentimiento de confianza, admiración, lealtad, adhesión y respeto hacia el líder,

\section{Figura 1}

\section{Aspectos asociados al liderazgo carismático}

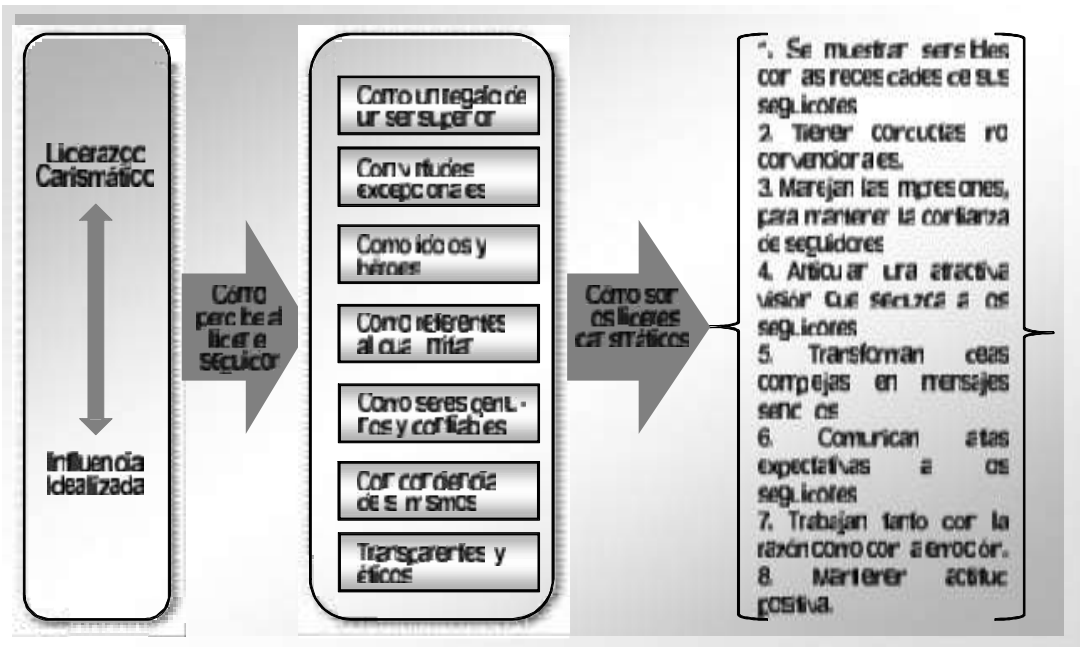

Fuente: Elaboración propia, basado en Parke y Wormell (1956); Choza (1993); Sampson (1997); Alvira (2000); Freixa (2003); Masciotra (2005); Fierro (2005); Kernis y Goldman (2006); Fernández (2006); Moriano et al (2011); Gil (2011); Vignale (2012); García-Calvo (2012). 
y están motivados para hacer incluso más de lo que en un principio se esperaba de ellos. Este fenómeno se describe como un proceso de influencia que subyace a seguidores fuertemente implicados, que son más conscientes de la importancia de su resultado en las tareas específicas que desarrollan; por lo mismo, habitualmente trascienden y postergan sus propios intereses por el bien de la organización.

Un líder transformacional es reflexivo, proactivo, dinámico y persuasivo, siendo capaz de generar conciencia en sus seguidores para el logro de metas colectivas y resultados notables; en este sentido, este enfoque comprende cuatro factores claves, los que pueden apreciarse en la Figura 2.

Los elementos de la Figura 2, están contenidos en el cuestionario "Multifactor Leadership Questionnaire" (MLQ), que
Bernard Bass desarrolló el año 1985; este instrumento -válido para medir el comportamiento de un líder transformacional- es quizás la aportación más relevante y un factor clave para desarrollar investigaciones sobre liderazgo a nivel organizacional. El contenido de estra herramienta ha tenido mutaciones con el tiempo, y se han añadido nuevos comportamientos consoiderados transformacionales, en las versiones desarrolladas por Bass (1996) y Bass y Avolio (1990).

El Multifactor Leadership Questionnaire (MLQ), se administra a los colaboradores, quienes deben calificar la conducta de su líder a través de los cuatro factores (Figura 2), que a continuación se explican: (Bass et al., 2003; Yukl, 2006 y Nemanich y Keller, 2007).

a. El primer factor se denomina consideración individualizada y hace referencia al conocimiento pormenorizado

Figura 2

Factores claves del liderazgo transformacional

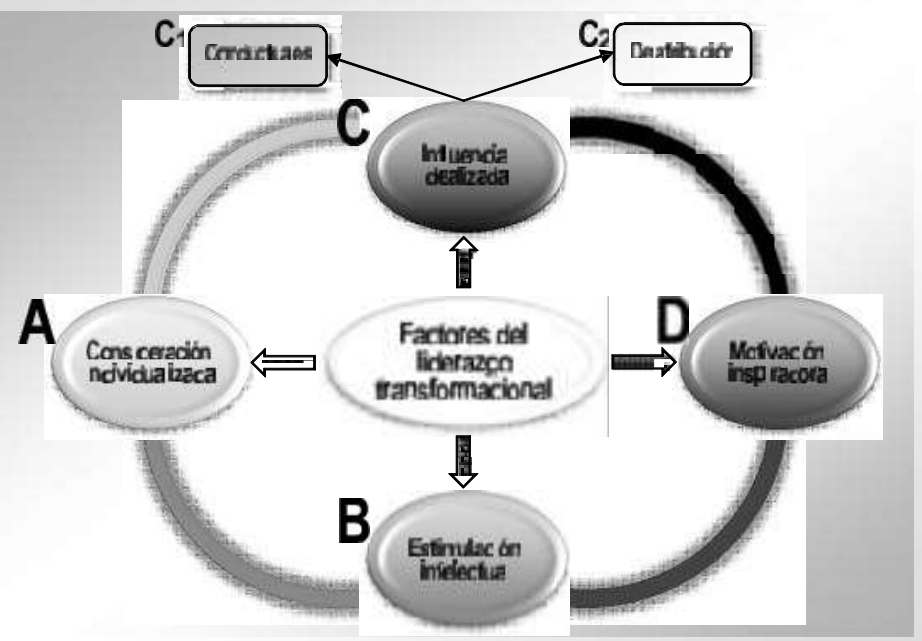

Fuente: Elaboración propia, basado en Bass (1998 y 1999). 
de cada necesidad particular de sus colaboradores. Esto permite diseñar estrategias individuales de soporte y bienestar a cada trabajador, dado que se reconocen sin problemas las disimilitudes que presentan las personas. Se sostiene que lo ideal es implementar un proceso y establecer un funcionamiento que evite que los seguidores se sientan supervigilados $y$ permanentemente evaluados. Este componente también hace referencia al rol del mentor o entrenador para la identificación y el desarrollo de potenciales líderes.

b. El segundo factor es la estimulación intelectual. Este aspecto considera la actitud del líder, que alienta a sus colaboradores a esforzarse, a ser diligentes, creativos, con capacidad para cuestionar supuestos, redefinir problemas y afrontar primitivas situaciones de manera más innovadora. Un elemento clave en esta parte, es la inexistencia de críticas públicas, respecto de los eventuales errores que puedan cometer las personas en el desempeño de sus funciones. De esta manera los líderes hacen cumplir a sus seguidores las actividades necesarias para el buen funcionamiento de la organización, logrando que ellos sean capaces de respetar y alinearse a las creencias y valores institucionales.

c. El tercer factor, denominado influencia idealizada, hace referencia a los líderes que son admirados, bienquistos, reconocidos, respetados y valorados; características que le permiten obtener la confianza y el valimiento de sus seguidores. Estas cualidades convierten a estos líderes transformacionales en ejemplos, en arquetipos a seguir, por parte de sus colaboradores. En este componente, se pueden distinguir dos sub-factores a saber:

c.1. Conductuales: hace referencia al comportamiento específico que el líder exhibe, el que genera una influencia positiva sobre sus seguidores.

c.2. De atribución: este sub-factor apunta a la descripción de aquellas actuaciones que provocan complacencia y fascinación en sus colaboradores.

d. Finalmente está el factor motivación inspiracional, se refiere a los líderes que son capaces de transmitir con convicción su motivación y pasión por lo que hacen; logrando con ello, alentar y estimular a sus colaboradores, hechos que proporcionan un significado a su trabajo. En estas circunstancias, se vigoriza el trabajo en equipo; así mismo, el líder tiene la obligación de formular una visión de futuro atractiva para los empleados y evidentemente para la organización.

Adicionalmente se reconoce que el líder transformacional influencia a sus seguidores estableciendo ciertas pautas conductuales que sirven de guía y ayudan a encauzar y direccionar esfuerzos para el logro de metas individuales y colectivas. Estos comportamientos se materializan en tres prácticas a saber: AUTOR

a. Visión compartida: esta situación ocurre cuando el líder transformacional es capaz de vislumbrar un punto claro al cual debe orientarse la organización; pero junto con identificar esa visión, debe tener la capacidad 
para encauzar a sus seguidores en esa dirección. Lo anterior significa que la visión compartida se logra tener, cuando existe en un grupo humano, una interpretación analógica y relativamente simétrica, respecto del punto al cual se quiere llegar (meta o puerto), gestando una especie de sentimiento homogéneo que empapa e imbuye toda la organización; brindando de esta forma, adherencia y coherencia a las diversas actividades que se realizan.

b. Pautas de referencias: los líderes transformacionales están disponibles y dispuestos a correr riesgos, pera también a seguir caminos diferentes para lograr sus propósitos organizacionales; en este sentido, se requiere de marcos referenciales que establezcan patrones y lineamientos conductuales que guíen y enfoquen el acionar de los diversos actores al interior de una organización.

c. Administración de impresiones: el líder transformacional tiene la capacidad para planificar, organizar, operacionalizar y utilizar estrategias y tácticas tendientes a visibilizar y resaltar su atractividad y las razones por las cuales puede cautivar y encantar a sus seguidores. Desarrolla acciones tendientes a involucrar e inspirar a sus colaboradores hacia los propósitos cardinales previamente definidos; teniendo presente que lo importante es precintar y posicionar su impronta en la mente de su personal.

Por otra parte, Rafferty y Griffin (2004) describieron el liderazgo transformacional como un estilo que proporciona una visión, reconocimiento y contribución para estimular la inteligencia de sus seguidores. Por lo tanto "Iíderes y seguidores se interrelacionan, para alcanzar niveles elevados de moral y motivación" (Burns, 1978). Los líderes que poseen como característica la influencia idealizada tendrán mayor disposición a involucrarse en actividades laborales de mayor riesgo y, por lo tanto, serán más influyentes, eficaces y con una mayor disposición a confiar en sus empleados (Bass, 1990; Bass y Riggio, 2006). Además, estos líderes otorgan una mayor autonomía y confianza hacia sus seguidores para llevar a cabo tareas y tomar de decisión que promuevan comportamientos innovadores en los empleados (Scott y Bruce, 1994; Conger y Kanungo, 1988).

La Figura 3, recoge sintéticamente los elementos más característicos del liderazgo transformacional, colocando el foco en la forma como estos líderes transforman a sus seguidores en líderes autodirigidos.

En cuanto al comportamiento del líder, Avolio y Bass (1991) y Bass (1998), analizan la conducta de éste en un cuadrante que relaciona y vincula cuatro variables; por un lado, la eficacia (que va verticalmente de efectivo a inefectivo) y por otro, su nivel de actividad (que se mueve horizontalmente de pasivo a activo). Lo autores precitados, sostienen que las relaciones de las variables dan como resultado dos perfiles:

a. En primera instancia, y según lo que se puede observar en la Figura 4, se identifica al líder con un perfil óptimo, que de menos a más (en una escala de 1 a 5) frecuentemente muestra las siguientes conductas:

a.1. De "Laissez-Faire" (LF), 


\section{Figura 3}

Características más importantes del liderazgo transformacional

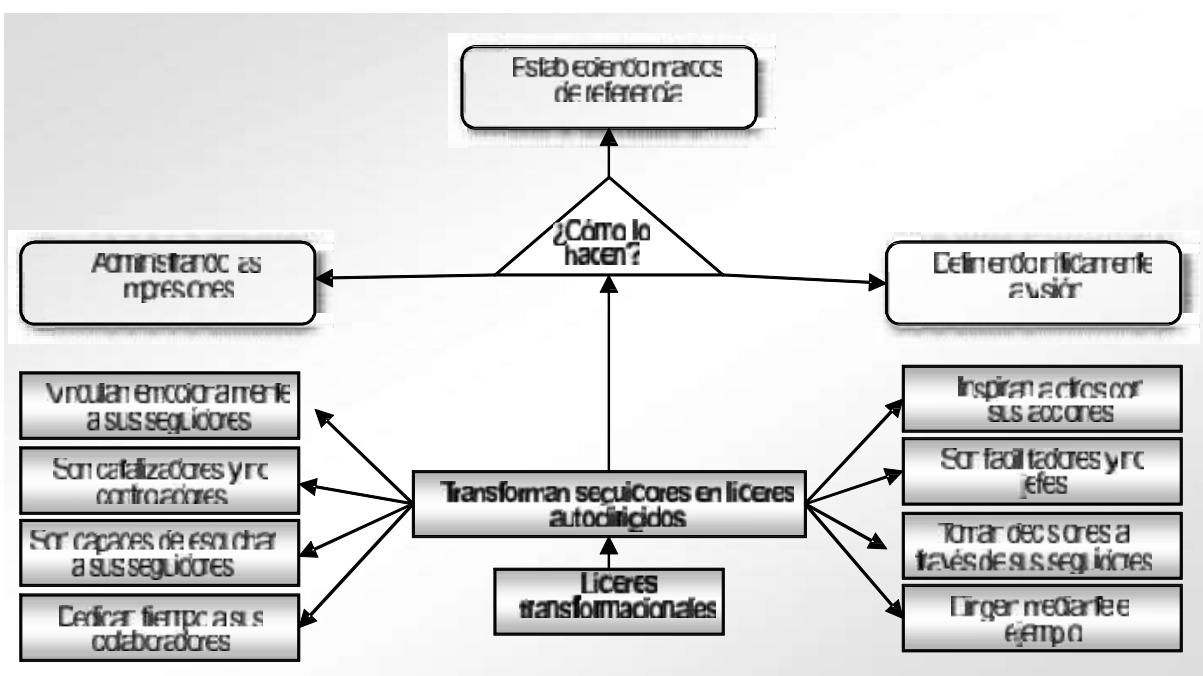

Fuente: Elaboración propia, basado en Bass (1985, 1990, 1998); Conger y Kanungo (1988); Scott y Bruce (1994); Rafferty y Griffin (2004); Bass y Riggio (2006).

\section{Figura 4 \\ Perfil óptimo del liderazgo}

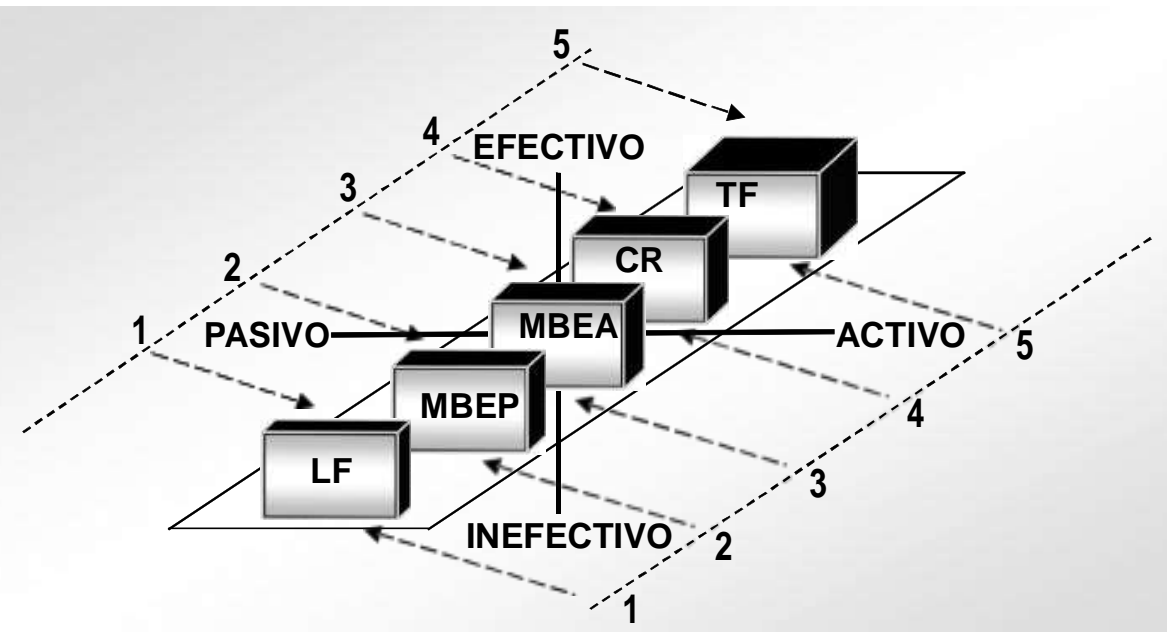

Fuente: Bass (1998). 
a.2. De dirección por excepción pasiva (MBEP),

a.3. De dirección por excepción activa (MBEA),

a.4. De recompensa contingente $(\mathrm{CR})$,

a.5. De Liderazgo transformacional.

b. En segundo término, y tal como puede visualizarse en la Figura 5, se grafica el liderazgo desde la mirada de un perfil sub-óptimo. En este caso, los sujetos humanos exhibirían la frecuencia de conductas de forma inversa al perfil óptimo, esto quiere decir que la ubicación de las variables presenta el siguiente orden:

b.1. De liderazgo transformacional,

b.2. De recompensa contingente,

b.3. De dirección por excepción (activa),

b.4. De dirección por Excepción (pasiva),

b.5. De "Laissez-Faire".

\section{Investigaciones sobre liderazgo}

Otra cuestión relevante, que es necesario considerar, dice relación con el hecho cierto de que una gran parte de la investigación contemporánea sobre liderazgo se ha centrado en los efectos del Iiderazgo transformacional y carismático sobre la motivación, el rendimiento de los seguidores y por supuesto, la eficacia del líder (Bass, 1985; Bass y Goodheim, 1987; Hater y Bass, 1988; Seltzer y Bass, 1987; Waldman et al.,1987; Waldman et al., 1990; Avolio, 1999; Bass y Avolio, 1994, 1997; Conger y Kanungo, 1988; Lowe y Gardner, 2000).

Como ya ha quedado de manifiesto, el instrumento más ampliamente utilizado para evaluar dichos efectos en las organizaciones es el denominado Multifactor Leadership Questionnaire (MLQ)

Figura 5

Perfil sub-óptimo del liderazgo

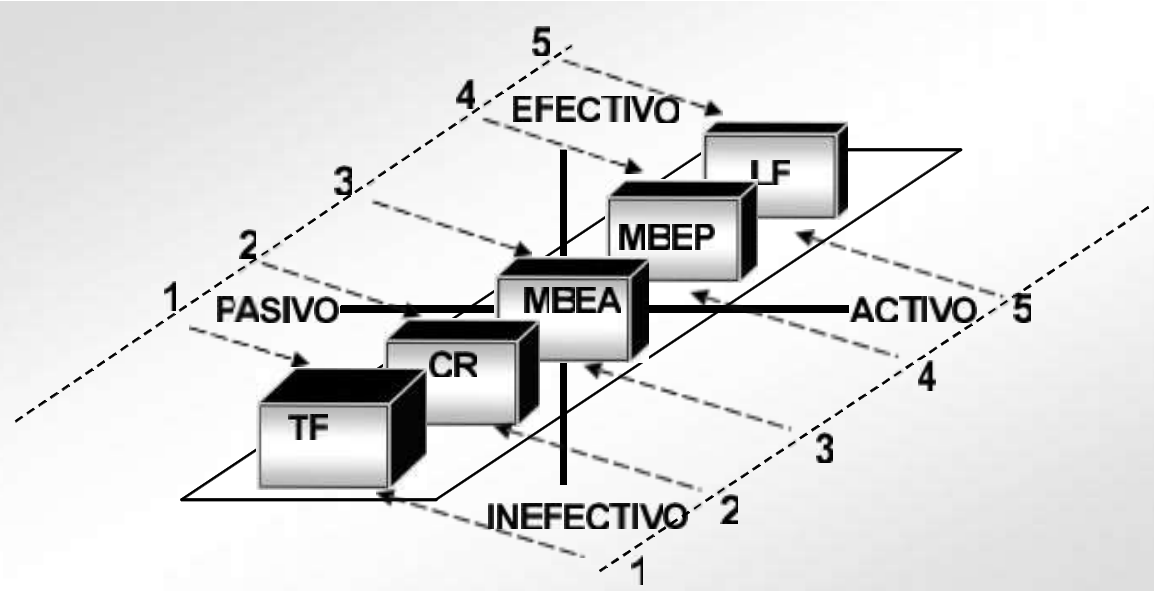

Fuente: Bass (1998). 
(Hunt, 1999; Lowe et al.,1996; Yukl, 1999), esta herramienta ha permitido detectar que el líder transformacional muestra correlaciones más altas y positivas que el líder carismático, particualrmente en cierto criterios como: eficacia y satisfacción de sus colaboradores. De esta forma, muchos autores han aseverado, a partir de sus indagsciones, que el liderazgo transformacional aumenta el compromiso e implicación de los trabajadores (Becker y Billings, 1993; Koh, 1990; Niehoff et al.,1990), la lealtad (Fullagar et al., 1992), y también el desempeño de los seguidores, ayudando a gestionar situaciones de estrés. Por su parte, se cree que el liderazgo carismático induce en mayor medida el estrés (Bass, 1999).

Existen también diversos meta-análisis (Gasper, 1992; De Groot et al., 2000; Dumdum et al., 2002; Lowe et al., 1996; Patterson et al., 1995) que confirman estos resultados. Más recientemente, Judge y Piccolo (2004) realizaron un meta- análisis, llegando a resultados similares a las líneas de trabajos anteriores (Lowe et al., 1996); pero, con valores menos contundentes y significativos (Fromm, 2005). Asimismo, la evidencia señala que la relación de estas variables con el factor transaccional de recompensa contingente también es positiva pero menor. Por el contrario, la asociación de la eficacia y la satisfacción con los estilos de liderazgo pasivos (laissez-faire o dirección por excepción pasiva) es muy negativa. Además, se reafirma en estudios posteriores que el Liderazgo Transformacional es más efectivo que la recompensa contingente, y ésta es más efectiva que la dirección por excepción, siendo el menos efectivo el "Liderazgo Carismático",
También resulta interesante referirse a la evidencia existente respecto de la perspectiva género; en este orden de cosas, Eagli et al (2003) llevaron a cabo un meta-análisis, centrándose en las diferencias que se pueden percibir dependiendo si se trata de damas o varones. Los resultados de sus trabajos investigativos demostraron que las mujeres son más transformacionales que los hombres en su estilo de liderazgo, admitiendo una fuerte influencia en roles muy influidos por expectativas sexistas.

Bass (1985) reflexiona que las teorías existentes sobre el liderazgo, se han centrado principalmente en la aclaración de roles y objetivos del seguidor, así como también en las formas de recompensar y sancionar el comportamiento de los subordinados (lo anterior, es más propio de un estilo de Liderazgo Transaccional, que se limita solamente al intercambio entre un líder y sus seguidores). Es por ello, que Bass (1985) sugiere un cambio de paradigma, que ayude a entender como los líderes influyen sobre sus seguidores al momento de trascender su interés propio por el bien de su organización, con la finalidad de alcanzar niveles óptimos de rendimiento. La comprensión de este fenómeno es facilitada por el liderazgo transformacional. En relación al perfil de este líder transformacional, el mismo Bass (1998) propone una orientación para estos líderes, en una lógica contoingencial o situacional, lo que implica atribuirle opciones de ductilidad, indicando que un estilo $u$ otro pueden ejercerse desde cualquier orientación, verbi gracia: transformacional-directivo o transformacional-participativo. De alguna forma, es lo que intenta recoger el Cuadro 1, haciendo una comparación descriptiva entre lo participativo y lo colectivo (directivo). 


\section{Cuadro 1}

\section{Descripciones de liderazgo participativo frente a directivo}

\begin{tabular}{|c|c|c|}
\hline & Participativo & Colectivo \\
\hline Lasset-fairs & $\begin{array}{l}\text { Cublquier cos que piemes como } \\
\text { correcta, también lo será para ml }\end{array}$ & 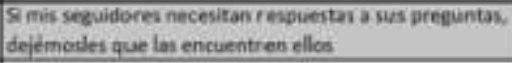 \\
\hline Dirección par excepción & $\begin{array}{l}\text { Vamos a desarrollar fartos las rejglas } \\
\text { que usaremos para ideriticar } \\
\text { errares. }\end{array}$ & $\begin{array}{l}\text { Estim son las reelas, y esta es la forma en que usted las } \\
\text { ha violado }\end{array}$ \\
\hline Aecompensa contingente & $\begin{array}{l}\text { Pongamonos de acuerdo en lo que } \\
\text { debe ser hecho y cóno verd } \\
\text { tecompensado al canse guirlo }\end{array}$ & $\begin{array}{l}9 \text { iated consigue los nbietivos que ha marcado, } \\
\text { reconocere su lagro con la siguiente recempensa }\end{array}$ \\
\hline $\begin{array}{l}\text { Consideración } \\
\text { individualisada }\end{array}$ & $\begin{array}{l}\text { CQué podemos como erupe para dar } \\
\text { a cada uno el apoyo necesario para } \\
\text { desarrallar sus capscidades }\end{array}$ & $\begin{array}{l}\text { Le voy a proporcionar el apoyo que necresita en sus } \\
\text { esfuerabs de austo decarrollo en el trabajo }\end{array}$ \\
\hline Estimulacion intelectual & $\begin{array}{l}\text { Podemos intentar reviser aluestras } \\
\text { ideas come grupo, sin ser craticos con } \\
\text { Las ideas de for demas hasta que } \\
\text { todes hayan sido tenidas en cuents }\end{array}$ & $\begin{array}{l}\text { Usted debe reesaminar la idea de que un motor de } \\
\text { fuvón fria es fiscamente imposible. Retome ese } \\
\text { problema y cuesticnese esa idea }\end{array}$ \\
\hline Motivacón inspiracional & $\begin{array}{l}\text { Vamos a trabajra juntos para unif } \\
\text { nuestras asperaciones y metas por ef } \\
\text { bien de nuestro grupa. }\end{array}$ & $\begin{array}{l}\text { Usted necesita decirse a si mismo que cada dla es } \\
\text { mejor, Debe fajarse en su progresión y continoar } \\
\text { sreciendo en el future }\end{array}$ \\
\hline Influencia idealizada & $\begin{array}{l}\text { Podemos ser un equipo ganador por } \\
\text { nuestra fe en nuestro compaliero. } \\
\text { Necesito vuastro apoyo para lograr } \\
\text { nuestra misión }\end{array}$ & 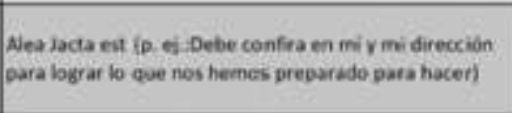 \\
\hline
\end{tabular}

Fuente: Bass (1998).

Bass y Avolio (1997), realizan un aporte muy sustantivo en los estudios de liderazgo, dado que incorporan en sus indagaciones, aquellas variables o cambios que privilegiarán la calidad y cantidad en el desempeño, la sustitución de objetivos o metas, la implementación de decisiones bajo contextos específicos y la resistencia al cambio. Lograr estas mutaciones, por medio de nuevas culturas, principios y valores, es quizás, el principal propósito de la organización moderna, con miras a obtener mejoras, que signifiquen una mayor creación de valor y por consiguiente un rendimiento sobresaliente. En esta misma línea argumental, Naisbitt (1994) indica que a través de líderes con ideas innovadoras, se propiciaran instancias que fortalecerán la innovación, creatividad y excelencia, para así de esta manera, aumentar la competitividad de las organizaciones.
Como ha quedado de manifiesto, el liderazgo transformacional puede cambiar 0 adaptarse a los escenarios intraorganizacionales adversos, para beneficiar no sólo a los individuos que laboran en la institución, sino que a toda la organización. Este líder debe ser capaz de producir una variación o transformación profunda en la apreciación que sus colaboradores tienen de él. Esta variación en la perspectiva, puede variar de contexto en contexto y de tarea a tarea. En general, se caracterizará por una modificación fundamental en ambas configuraciones y en la comprensión del problema concreto al que se enfrentan los colaboradores. Para cambiar rápidamente, las personas deben estar preparadas para desarrollar ideas fuera de su "zona confortable" y en conflicto con "verdades absolutas", repetidas y desgastadas. Esta mutación asincró- 
nica requiere un cambio de perspectiva, la cual es promovida de manera más nítida por el liderazgo transformacional. Kuhnert y Lewis (1987) se refieren a este cambio como al sistema de "creación de significado" o cómo los individuos interpretan los retos ante ellos en sus trabajos y carreras.

El liderazgo puede ayudar a potenciar las necesidades de madurez de los colaboradores y estos a su vez, las del líder. Esto trae como consecuencia un mayor interés en potenciar las características de desempeño y desarrollo a nivel grupal. Para cambios más intensos, profundos y de mayor grado, se requieren factores propios de un líder transformacional, el cual debe tener la capacidad para propiciar un cambio de orientación a nivel de proceso y de resultados con implicaciones en el mediano y largo plazo.

En consecuencia, el líder transformacional se caracteriza -entre otras cosas- porque es capaz de articular una visión compartida sobre el futuro, estimulando intelectualmente a sus seguidores, y prestando gran atención a las diferencias individuales de los sujetos que son parte de una organización determinada (Yammarino y Bass, 1990).

Así el liderazgo transformacional, puede traer como consecuencia un incremento a niveles superiores del rendimiento de los seguidores, estableciendo motivadores y formulando acciones claras para el cumplimento de las metas por parte de los subordinados. Esta capacidad del líder transformacional para obtener un rendimiento superior, más allá de las expectativas individuales de sus colaboradores, se ha denominado la "Hipóte- sis de Aumento" (Waldman et al., 1987; Waldman et al., 1990).

En esta misma línea, se han desarrollado investigaciones, que han llegado a las mismas conclusiones, considerando variados contextos y niveles de análisis, como por ejemplo:: en organizaciones público-privadas, en altos y bajos niveles de responsabilidad $y$, en diferentes niveles educacionales de los líderes, (Avolio et al.,1991; Bryce, 1989; Keller, 1992; Waldman et al., 1990; Cowen, 1990; Koh et al., 1990; Seltzer y Bass, 1987; Bass, 1985; Avolio y Howell, 1992; Salter, 1989; Young, 1990; Komives, 1991; Ross, 1990; Bryant, 1990). Estos hallazgos sugieren que el liderazgo transformacional provoca efectos a nivel integral, recursivo y departamental en una organización (Howell y Avolio,1993). Además, esta evidencia demuestra que obteniendo niveles altos de liderazgo transformacional, se acrecienta la varianza explicativa de otros estilos de liderazgo para predecir otras variables de resultado; por ejemplo: "Performance", "Cumplimiento de Metas", "Evaluación de la eficacia", "Esfuerzo Extra de Superiores y Subordinados", "Efectividad en el Desempeño del Trabajo", "Eficacia", "efectividad de la Unidad y "Satisfacción".

\section{Liderazgo carismático y liderazgo transformacional}

La investigación en el campo de la administración de organizaciones, ha enfrentado por años el desafío de esclarecer las diferencias y similitudes entre el comportamiento efectivo de un líder tansformacional y un líder carismático. Esta situación, ha acrecentado la ambigüedad y la carencia de coherencia metodológica en su terminología y medición, lo que difi- 
culta aún más los parangones que se puedan realizar entre estos dos estilos o formas de asumir el liderazgo. Este hecho no impide mencionar, que un líder transformacional parece más proclive a tomar medidas que convierten a sus seguidores en socios estratégicos, para la consecución d las metas grupales. En cambio, un líder carismático tiende a hacer hincapié en la necesidad de una mutabilidad radical que sólo se puede lograr si los seguidores confían plenamente en la experiencia e idoneidad de su líder. Lo analizado y comentado en este escrutinio, apoya la idea de que el liderazgo transformacional emerge como una fórmula ideal y adecuada para desarrollar genuinamente las organizaciones; pero junto con ello, se reconoce el impacto y la ascendencia que puede lograr el liderazgo carismático.

Por otra parte, Bass y Avolio (1993), han sustentado ue el liderazgo transformacional difiere del liderazgo carismático, ya que este último, es sólo una dimensión del primero, vale decir, el carisma es necesario pero no suficiente para conseguir un liderazgo transformacional. Sin embargo, existen algunos que tienden a asentir, que se puede ser carismático sin ser transformacional; otros desde su mirada, no están totalmente de acuerdo con esta aseveración, pues colocan el acento en la importancia de un carisma excepcional y sobresaliente en el liderazgo (Conger y Kanungo, 1987, 1994; House y Shamir, 1993), y por lo mismo, es factible inferior que se debería buscar una mixtura apropiada de estas dos perspectivas.

En este sentido y a modo de estribación teórica sumaria, es factible expresar de forma límpida, que dada las evidencias hemerográficas y también empíricas consultadas, no es factible separar fácilmente el liderazgo transformacional, del carismático; como tampoco es posible afirmar que uno es peor o mejor que otro; si se podría asentir que los dos tipos se complementan y son esenciales para el logro de los propósitos organizacionales; la efectividad de uno u otro, dependerá de la correctas aleación que se realice, el tipo de sujeto humano que lo utilice y el escenario intra $y / 0$ periorganizacional que tenga la respectiva institución.

Como corolario definitivo, en el Cuadro 2 se presentan las dos teorías o enfoques analizados, identificando a sus autores más representativos; adicionalmente se incorpora una columna que identifica las conclusiones más importantes.

\section{Cuadro 2 \\ Estilos de Liderazgo}

\begin{tabular}{|c|c|c|}
\hline Teoría & Autor & Principal conclusión \\
\hline \multirow[t]{3}{*}{$\begin{array}{l}\text { Liderazgo } \\
\text { Carismático }\end{array}$} & \multirow{3}{*}{$\begin{array}{l}\text { House (1977), } \\
\text { Conger y Ka- } \\
\text { nungo (1987) }\end{array}$} & $\begin{array}{l}\text { Se define en términos de la influencia del líder sobre sus seguidores y la naturaleza de la relación lí- } \\
\text { der-seguidor. }\end{array}$ \\
\hline & & El líder busca que adopten su visión, pero que no la cuestionen. \\
\hline & & Siguen al líder de manera "ciega", dado que se le considera que tienen un don, o una dimensión espiritual. \\
\hline \multirow{3}{*}{$\begin{array}{l}\text { Liderazgo } \\
\text { Transfor- } \\
\text { macional }\end{array}$} & \multirow{3}{*}{$\begin{array}{l}\text { Burns } \\
(1978), \text { Bass } \\
(1985)\end{array}$} & $\begin{array}{l}\text { Implica un proceso de influencia subyacente que motiva a los seguidores animándoles a superar sus } \\
\text { propios intereses en aras de la organización. }\end{array}$ \\
\hline & & El líder impregna su visión, pero genera condiciones para que pueda ser discutida e incluso cuestionada. \\
\hline & & $\begin{array}{l}\text { Están motivados para hacer más de lo esperado y sentir confianza, lealtad, respeto y admiración ha- } \\
\text { cia el líder. }\end{array}$ \\
\hline
\end{tabular}

Fuente: Elaboración propia basado en House (1977); Conger y Kanungo (1987); Burns (1978); Bass (1985). 


\section{Conclusión}

Este trabajo se inició con un acercamiento al concepto de liderazgo, para dar paso a una revisión de dos ópticas o enfoques complementarios, como lo son los del liderazgo carismático y el del liderazgo transformacional.

Se colige que el liderazgo transformacional ejerce una influencia mayor a nivel de cultura o desarrollo organizacional, en cambio el liderazgo carismático está centralizado más bien en el líder visto como una figura espiritual acentuada en la persona, más que en el proceso de liderazgo, planteado por el estilo transformacional. En este orden de cosas, para entornos turbulentos, intrincados y dinámicos -donde las organizaciones deben estar constantemente en preparadas para adaptarse a diversas situaciones y contigencias, sean éstas de orden políti$\mathrm{co}$, social, cultural y/o económico- el liderazgo transformacional pareciera ser el más idóneo, por su focalización en los procesos o etapas a través de los que el equipo de trabajo se desarrolla y evoluciona. Sin embargo, se ha expresado de forma suplementaria, que dependiendo de las circunstancias, no es factible asentir a cabalidad que un estilo es mejor que otro, ni tampoco, que se trata de ópticas antagónicas; inclusive, se ha señalado que pueden complementarse adecuadamente; en definitiva, al parecer el valor radica en la pertinencia y congruencia con la cual se decide la utilización de uno u otro, puesto que una apropiada mixtura o jerarquización, posibilitará el mejor cumplimiento de las metas de una determinada organización.
Se espera que esta revisión proporcione una hoja de ruta para seguir avanzando en las teorías del liderazgo, en lo referente a incursionar y auscultar nuevas líneas de investigación tanto teóricas como empíricas, permitiendo así a las organizaciones comprender más plenamente los elevados niveles de rendimiento sostenido y verdadero, que un apropiado y pertinente proceso de liderazgo puede asegurarle a las diversas instituciones. Es innegable que a nivel latinoamericano se necesitan más estudios -especialmente empíricos- en estas temáticas, pues la cultura general, la cultura organizacional particular y la forma de ser de las personas, es muy distinta a la de los trabajadores norteamericanos 0 europeos (que son los continentes que generan el mayor nivel de conocimiento en estos tópicos).

En un contexto organizacional altamente globalizado y competitivo, donde la eficiencia, eficacia, efectividad, velocidad de respuesta y orientación hacia el cliente o usuario, se han transformado en verdaderos imperativos estratégicos, resulta casi axiomático, pero aun así necesario, plantear la relevancia de centrarse en el único factor que puede generar verdaderas ventajas competitivas nítidas y sostenibles en el tiempo: nos referimos evidentemente a las personas. En este sentido, el uso apropiado del talento laboral disponible, depende primordialmente de los estilos de liderazgo que tienen las personas que cumplen el rol de directivos o gerentes. Reflexionar sobre esta materia e indagar acerca de sus tendencias, tiene en si mismo un valor para el futuro, dado que posibilita prospectar la viabilidad de las organizaciones. 


\section{Referencias Bibliográficas}

Alvira, Rafael (2000) ¿Qué significa "buena voluntad?. Anuario Filosófico (33), pp. 723-734.

Antonakis, John; Avolio, Bruce y Sivasubramaniam, Nagaraj (2004). Context and leadership: an examination of the nine-factor full-range leadership theory using the Multifactor Leadership Questionnaire. The Leadership Quarterly, Vol.14, pp 261-295.

Avolio, Bruce (1999). Full leadership development: building the vital forces in organizations. Thousand Oaks, CA: Sage Publications

Avolio, Bass; Yammarino, Francis y Bass, Bernard. (1991). Identifying common methods variance with data collected from a single source: An unresolved sticky issue. Journal of Management, Vol. 17, no 3, pp 571-587.

Avolio, Bruce y Bass, Bernard. (1991). The Full Range leadership development programs: basic and advanced manuals. Binghamton. NY: Bass, Avolio y Associates.

Bass, Bernard (1985), Leadership and Performance Beyond Expectations. New York: Free Press.

Bass, Bernard (1996). Anew paradigm of leadership: An inquiry into transformational leadership. Alexandria, VA: U. S. Army Research Institute for the Behavioral and Social Sciences.

Bass, Bernard (1998). Transformational leadership: industrial, military, and educational impact. Mahwah, $\mathrm{NJ}$ : Erlbaum.

Bass, Bernard (1999). Two Decades of Research and Development in Transformational Leadership. European Journal of Work and Organizational Psychology, Vol. 8, pp 9-26.
Bass, Bernard y Avolio, Bruce (1990). Transformational Leadership development: Manual for the Multifactor Leadership Questionnaire. Palo Alto: Consulting Psychologists Press Inc.

Bass, Bernard y Avolio, Bruce (1993). Transformational Leadership: A response to critiques. En M. M. Chamers y R. Ayman (Eds.), Leadership: Theory, perspectives and direction. San Diego, CA Academic Press pp. 49-80.

Bass, Bernard y Avolio, Bruce (1994). Improving organizational effectiveness through transformational leadership. Thousand Oaks, CA: Sage Publications

Bass, Bernard y Avolio, Bruce (1997). Revised Manual for the Multifactor Leadership Questionnaire. Palo Alto, C. A.: Mind Garden.

Bass, Bernard, Avolio, Bruce y Goodheim, Laurie (1987).Biography tle assessment of transformational leadership at the world - class level. Journal of management, Vol. 13, $\mathrm{n}^{\circ}$ 1, pp. 7-19

Bass, Bernard; Avolio, Bruce; Jung, Dong y Berson, Yair (2003). Predicting Unit Performance by Assesing Trasnsformational and Transactional Leadership. Journal of Applied Psychology, Vol. 88, No.2, pp. 207-218.

Bass, Bernard y Riggio, Ronald (2006). Transformational leadership (2nd ed.). Mahwah, NJ: Lawrence Erlbaum Associates.

Becker, Thomas y Billings, Robert (1993). Profiles of commitment: An empirical test. Journal of organizational behavior, $\mathrm{n}^{\circ} 14$, pp. 177-190.

Bryant, Allan (1990). Relationship between nurse managers perceived transformational versus transactional leadership styles and staff nurse turnover. Unpublished masters thesis, University of Akron, Akron, $\mathrm{OH}$. 
Bryce, Yokochi (1989). Leadership styles of Japanese business executives and managers: Transformational and transactional (Doctoral dissertation, U.S. International University). Dissertation Abstracts International, 5006B.

Burns, James (1978). Leadership. New York: Harper y Row.

Casado, José (2003). El valor de la persona: nuevos principios para la gestión del capital humano. Pearson Educación de Chile Ltda.

Castro, Alejandro y Lupano, María (2005). Diferencias individuales en las teorías implícitas del liderazgo y la cultura organizacional percibida. Boletín de Psicología, No. 85, pp. 89-109.

Certo, Manuel (2000). Administración moderna.Pearson Educación de Colombia, Bogota. $8^{\circ}$ edición.

Conger, Jay y Kanungo, Rabindra (1987) Charismatic leadership: the elusive factor in organizational effectiveness. San Francisco: Jossey-Bass.

Conger, Jay y Kanungo, Rabindra (1988). Toward a behavioral theory of charismatic leadership in organizational settings. Academy of Management Review, Vol. 12, pp. 637-647.

Conger, Jay y Kanungo, Rabinda (1994). Charismatic leadership in organizations: Perceived behavioral attributes and their measurement. Journal of Organizational Behavior, Vol 15, pp. 439-452.

Cowen, Scott (1990). A study of relationships between perceived leader behaviors of presidents at public four-year institutions of higher education in the United States and the changes in FTE enrollment, perceptions of effectiveness, subordinate satisfaction, and other factors of the presidency (Doctoral dissertation, Gonzaga University). Dissertation Abstracts International, 5301A.
Choza, Jacinto. (1993). Las máscaras del sí mismo. Anuario Filosófico (26), pp. 375-394

DeGroot, Timothy; Kiker, Scott y Cross, Thomas (2000). A meta-analysis to review organizational outcomes related to charismatic leadership. Canadian Journal of Administrative Sciences, Vol. 17, pp. 356-371.

Diccionario de la Real Academia Española (RAE 2014). http://www.rae.es/obrasacademicas/diccionarios/diccionario-de-la-lengua-espanola

Dumdum, Rex; Lowe, Kevin; Avolio, Bruce y Bass, Bernard. (2002). A meta-analysis of transformational and transactional leadership correlates of effectiveness and satisfaction: An update and extension (pp. 36-66). In Bass. James. Avolio y F. J. Yammarino (Eds.), Transformational and charismatic leadership: The road ahead. Oxford, U.K.: Elsevier Science

Eagly, Alice; Johannesen-Schmidt, Mary y Van Engen, Marloes (2003). Transformational, transactional, and laissez-faire leadership styles: a meta-analysis comparing women and men. Psychological bulletin, Vol. 129, no 4, pp. 569-591

Fernández, Guadalupe (2002). Talento Directivo: cómo medirlo y desarrollarlo. Pearson Educación, S.A., Madrid

Fernández, Domingo (2006). Foucault, identidad y sexualidad. Revista de Filosofía A Parte Rei $n^{\circ} 45$

Fierro, Alfredo (2005). Uno mismo a examen. Escritores de Psicología. $N^{\circ} 7 \mathrm{pp}$ 15-23

Freixa, Esteve (2003). El conocimiento de sí mismo desde la óptica conductista. Vol. Revista Psicothema, Vol. 15, $\mathrm{n}^{\circ}$ 1, pp. 1-5

Fromm, Michael (2005). Transformational and Transactional Leadership: A MetaAnalytic Test of Their Relative Vali- 
dity. Leadership Review, Vol. 5, p. 113-115.

Fullagar, Clive; McCoy, Don y Shull, Carla (1992). The socialization of union loyalty. Journal of Organizational Behavior, Vol. 13, p. 13-26.

Ganga, Francisco y Navarrete, Erwin. (2013). Enfoques asociados al liderazgo eficaz para la organización. Revista Gaceta Laboral. Vol. 19. № 1.

Gasper, Sandra (1992). Transformational leadership: An integrative review of the literature. Doctoral Dissertation. Kalamazoo, Ml: Western Michigan University.

García-Calvo, Tomás. (2012). Procesos grupales y su relación con el rendimiento deportivo. CCD 21-Suplemento 1, vol. 7.

Gil, Francisco et al (2011). Nuevas formas de liderazgo en equipos de trabajo. Papeles del Psicólogo. Vol. 32 (1), pp. 38-47.

Hater, John y Bass, Bernard (1988). Superiors evaluations and subordinates' perceptions of transformational and transactional leadership. Journal of Applied Psychology, Vol. 73, p. 695-702.

House, Robert (1977). A 1976 theory of charismatic leadership. En J.G. Hunt y L. L. Larson (Eds.), Leadership: The Cutting Edge, Carbondale: Southern Illinois University Press, pp.189-207.

House, Robert y Shamir, Boas (1993). Towards the integration of transformational, charismatic, and visionary theories. In M. M. Chemers \& R. Ayman (Eds.), Leadership theory and research: perspectives and directions. San Diego, CA: Academic Press.

House, Robert; Javidan, Mansour y Paul Hanges, Dorfman, Peter (2002). Understanding cultures and implicit leadership theories across the globe: an introduction to project GLOBE. Journal of World Business. Vol. 31. № 1. 3-10.
Howell, Jane y Avolio, Bruce (1993). Transformational leadership, transactional leadership, locus of control, and support for innovation. Journal of Applied Psychology, Vol. 78, pp. 891-902.

Howell, Jane y Avolio, Bruce (1993),.Transformational leadership, transactional leadership, locus of control, and support for innovation. Journal of Applied Psychology, Vol. 78, pp. 891-902.

Hunt, James (1999). Transformational/charismatic leadership's transformation of the field: an historical essay. The Leadership Quarterly, Vol.10, 129-144.

Jay A. Conger and Rabindra N. Kanungo (1987). Toward a Behavioral Theory of Charismatic Leadership in Organizational Settings. The Academy of Management Review. Vol. 12, No. 4 (Oct., 1987), pp. 637-647.

Judge, Timothy and Piccolo, Ronald (2004). Transformational and Transactional Leadership: A Meta-Analytic Test of Their Relative Validity. Journal of Applied Psychology 2004, Vol. 89, No. 5, 755-76

Keller, Robert (1992). Transformational leadership and the performance of research and development project groups. Journal ofManagement, $\mathrm{Vol}$ $18 N^{\circ} 3,489-501$.

Kernis, Michael, y Goldman, Brian. (2006), A multicomponent conceptualization of authenticity: Theory and research. In M. P. Zanna (Ed.), Advances in Experimental Social Psychology, Vol 38. (pp. 283-357). San Diego, CA: Elsevier Academic Press.

Koh, William. (1990). An empirical validation of the theory of transformational leadership o secondary schools in Singapore. Doctoral dissertation, University of Oregon, Eugene, OR. 
Koh, William; Steers, Richard y Terborg, James (1990). The effects of transformational leadership on teachers and students in Singapore. Paper presented at the annual meeting of the Academy of Management, Miami Beach, FL.

Komives, Susan (1991). The relationship of same- and cross- gender work pairs to staff performance and supervisor leadership in residence hall units. Sex Roles, Vol. 24, pp. 355-363.

Kuhnert, Karl y Lewis, Philip. (1987). Transactional and transformational leadership: A constructive developmental analysis. Academy of Management review, Vol. 12, pp. 648-657.

Lowe, Kevin; Kroeck, Galen y Sivasubramaniam, Nagaraj (1996). Effectiveness correlates of transformational and transactional leadership. A metaanalytic review of the MLQ literature. Leadership Quarterly, Vol. 7, pp. 385-425.

Lowe, Kevin y Gardner, Wendi (2000). Ten years of The Leadership Quarterly. contributions and challenges for the future. Leadership Quarterly, Vol 11, pp. 459-514.

Masciotra, Domenico (2005). Reflexibilidad, metacognición y competencia. Vie Pédagogique, Fevrier, $N^{\circ} 134$, pp. 29-31

Molero, Fernando, (1995), El estudio del carisma y del liderazgo carismático en las Ciencias Sociales: una aproximación desde la Psicología Social. Revista de Psicología Social, 10, 1, pp. 43-60.

Moriano, Juan et al. (2011). Liderázgo autentico. Concepto y validación del cuestionario ALQ en España. Psicothema, Vol. 23, n², pp.336-341

Naisbitt, John (1994). Global paradox: The bigger the world economy, the more powerful its smallest players. New York: W. Morrow.
Nemanich, Louise and Keller, Robert,( 2007). "Transformational leadership in an acquisition: A field study of employees", The Leadership Quarterly, Vol. 18, pp. 49-68.

Niehoff, Brian; Enz Cathy y Grover, Richard. (1990). The impact of top management actions on employee attitudes and perceptions. Group and Organizations Studies, Vol. 15, p. 337-352.

Patterson, Coleman et al (1995, April). A meta-analytic examination of leadership style and selected compliance outcomes. Paper presented at the 10th Annual Conference of the Society for Industrial and Organizational Psychology, Orlando, FL

Parke, Herbert y Wormell, Donald (1956). The Delphic Oracle. Volumen 1. Editor Blackwell.

Pirela, Ligia (2004). Enfoque epistemológico del liderazgo transformacional.Universidad de Zulia, Venezuela. Omnia, Vol. 10, No 2

Pirela de Faria, Ligia. (2010). Liderazgo y cultura organizacional en instituciones de educación básica. Revista Vnezolana de Gerencia, año 15, n 15.

Ramirez, Marbelis (2012). Estilos de liderazgo y sus enfoques gerenciales. Una aproximación teórica-metodológica para el análisis de la dirección organizacional. Revista de Ciencias Sociales, Vol. XVIII, Nº 1.

Rafferty, Alannah y Griffin, Mark (2004). Dimensions of transformational leadership: Conceptual and empirical extensions. The Leadership Quarterly, Vol.15, pp. 329-354.

Ross, Steven (1990). Transformational leadership: Measurement of personality attributes and performance effects on work groups (Doctoral dissertation, George Washington University). Dissertation Abstracts International, 5203B. 
Sampson, Anthony (1997). Lectura y cuidado de sí. Revista Universidad del Valle, Vol. 16, pp. 4-16.

Scott, Susanne and Bruce, Reginald (1994).The Academy of Management Journal. Vol. 37, No. 3, Jun.

Salter, Joseph (1989). Leadership styles in the United States marine corps transport helicopter squadrons. Naval Postgraduate School working paper.

Seltzer, Joseph y Bass, Bernard (1987). Leadership is more than initiation and consideration. New York, NY: American Psychological Association.

Robbins, Stephen, y DeCenzo, David (2008). Supervisión. Perason Educación, $5^{\circ}$ edic.

Trilling, Lionel (1972). Sincerity and Authenticity. Cambridge, NY: Harvard University Press.

Vignale, Silvana (2012) Cuidado de sí mismo y cuidado del otro. Aportes desde M. Foucault para pensar relaciobes entre subjetividad y educación. Revista Internacional de Filosofía, Vol. XVII, PP. 307-3024

Waldman, David; Bass, Bernard y Einstein, Walter (1987). Effort, performance and transformational leadership in industrial and military service. Journal of Occupational Psychology, Vol. 60, pp. 1-10.

Waldman, David; Bass, Bernard y Yammarino, Francis. (1990). Adding to contingent reward behavior: The augmenting effect of charismatic leadership. Group and Organizations Studies, Vol. $15 \mathrm{~N}^{\circ} .4$, pp. 381-391.

Yammarino, Francis y Bass, Bernard (1990). Long-term forecasting and of transformational leadership and its effects among naval officers: Some preliminary findings. In K.E. Clark and M.B. Clark (eds.), Measures of leadership pp. 151-169). West Orange, NY: Leadership Library of America.

Yammarino, Francis et al (2005). Leadership and levels of analysis: A state-of-thescience review. Leadership Quarterly, 16, 6, 879-919.

Young, Michelle (1990). Transformational leadership behaviors of male and female academic deans (Doctoral dissertation, Bowling Green State University). Dissertation Abstracts International, 52,082IA.

Yukl, Gary y Van Fleet, David (1992). Theory and research in organizations. En M. D. Dunnette y L. M. Hough (Eds.), Handbook of industrial and organizational psychology, 2nd ed., Vol. 2. Palo Alto, Ca: Consulting Psychologists Press, Inc.

Yukl, Gary (1999). An evaluation of conceptual weaknesses in transformational and charismatic leadership theories. The Leadership Quarterly, Vol. $10 \mathrm{pp}$. 285-305

Yukl, Gary (2006). Leadership in organizations, (6th ed.) Upper Saddle River, N. J.: Prentice Hall. 